\title{
Erratum to: The work of Douglas Munn and its legacy
}

\author{
John Fountain
}

Received: 28 October 2010 / Published online: 23 November 2010

(C) Springer Science+Business Media, LLC 2010

\section{Erratum to: Semigroup Forum (2010) 81:2-25 \\ DOI 10.1007/s00233-010-9240-3}

The definition given on page 15 for the Jacobson radical of a ring $R$ requires that $R$ has an identity. To include all rings we must define $J(R)$ to be the intersection of all modular maximal right ideals of $R$ [3] (see also [1] and [2] where modular right ideals are referred to as regular right ideals). A right ideal $I$ of a ring $R$ is modular if there is an element $e \in R$ such that $a-e a \in I$ for all $a \in R$.

On page 16, it is stated that simple rings are primitive. This assertion is true for rings with identity, but is, of course, well known to be false for general rings.

I am indebted to Professor H. E. Heatherly for drawing my attention to these errors.

\section{References}

1. Divinsky, N.J.: Rings and Radicals. University of Toronto Press, Toronto (1965)

2. Herstein, I.N.: Noncommutative Rings. Math. Assoc. of America, Washington (1968)

3. Jacobson, N.: Structure of Rings. Am. Math. Soc., Providence (1956)

Communicated by P. Silva.

The online version of the original article can be found under doi:10.1007/s00233-010-9240-3.

J. Fountain ( $₫)$

Department of Mathematics, University of York, Heslington, York YO10 5DD, UK

e-mail: jbf1@york.ac.uk 